\section{O2-02-03 DEVELOPMENT OF DEMQOL-U AND DEMQOL-PROXY-U: GENERATION OF PREFERENCE-BASED INDICES FROM DEMQOL AND DEMQOL-PROXY FOR USE IN ECONOMIC EVALUATION}

Sube Banerjee, King's College London, Institute of Psychiatry, London, United Kingdom.

Background: What is needed to enable cost-effectiveness evaluation in dementia? The currently available brief generic measures are problematic due to the error inherent in their use. We generated a preference-based single index for DEMQOL and DEMQOL-Proxy for use in economic evaluation. Objectives were: 1) to derive health state classification systems amenable to valuation from DEMQOL and DEMQOL-Proxy, 2) to generate utility values for every health state defined by the health state classification systems developed, and 3) to examine whether utility values elicited from the general population differ from utility values elicited from patients and carers for dementia. Methods: 1) Derivation of the health state classification system using item analysis and five separate Rasch models on DEMQOL ( $\mathrm{n}=$ $1189)$ and DEMQOL-Proxy $(\mathrm{n}=1223)$ data; 2$)$ General population valuation survey and modelling to produce values for every health state a general population survey with 600 subjects; 3) Patient/carer valuation survey - 71 people with dementia and 71 carers. Results: The factor structures were robust. Using stringent criteria we generated five items for DEMQOL and four for DEMQOL-Proxy. These new instruments were named DEMQOL-U and DEMQOL-Proxy -U. These descriptive systems were amenable to valuation. The population data were subject to multiple regression, from this two preference-based algorithms were estimated, one each for DEMQOL-U and the DEMQOL-Proxy-U, for valuing all dementia related states defined by these classifications. The models fitted to the data performed favourably compared to similar models estimated for other preference-based measures in terms of fit, prediction and the consistency of the coefficients. Conclusions: This means we can generate health state utility values from any dataset where one or both of DEMQOL and DEMQOL-Proxy have been completed. This is the first such dementia-specific system to have been developed and the first time that the values of the general population have been compared to patients and carers in this way. The values obtained from people with dementia and carers were systematically different to those from the general public, who tended to undervalue the impact of dementia. The population used to produce dementia health state utility values could impact upon the results of cost-effectiveness analysis and potentially affect resource allocation decisions.

\section{O2-02-04 EARLY INDICATORS OF DEMENTIA RISKS: TESTS OF COGNITION AND ACHIEVEMENT AMONG THE 1960 PROJECT TALENT HIGH SCHOOL COHORT}

James McNally, Martha Sayre, University of Michigan, Ann Arbor, Michigan, United States.

Background: The strength of existing studies of aging such as ELSA the HRS is their ability to grow and develop as approaches to measuring the cognition evolves. Challenges remain in that the measurement of dementia in most studies is done through proxy. Another challenge is that most studies of aging are based on a research approach that treats the elderly as an independent segment of the lifecourse. This paper examines the potential of a new study Project TALENT, was first fielded in the 1960's which is now prepared to reinterview its respondents 50 years later to see how early life events have impacted their later life outcomes. Methods: Project TALENT is the single largest source of information on the life experiences of students who were in high school in 1960. The data, acquired from more than 400,000 participants who were given a two-day battery of tests and inventories. The test battery was designed to provide baseline data on the aptitudes and abilities of these individuals, as well as to assess their interests and various personal characteristics. Results: The strength of existing studies of aging such as ELSA the HRS is their ability to grow and develop as approaches to measuring the cognition evolves but challenges In contrast, Project TALENT's strength is in the fact that they collected measures of cognition early, while the respondents were still in their teens. Now we have a powerful comparative basis to examine changes in cognition as the respondents enter their 60's and 70's. Conclusions: Project TALENT overcomes many if not all of the barriers faced by other studies that might be transitioned into life course studies of cognitive development and change.Aging does not begin at 50 , it begins at birth. While existing studies of aging represent invaluable resources and have provided immense contributions to our understanding of aging, research designs must move in new directions and we suggest that Project TALENT may be a useful direction to explore.

\section{O2-02-05 ORANGE COUNTY VITAL AGING PROGRAM: A COMMUNITY-BASED PROGRAM TO MAINTAIN BRAIN HEALTH AND REDUCE THE COSTS OF CARE FOR A POPULATION AT RISK FOR ALZHEIMER'S DISEASE}

William Shankle ${ }^{1}$, Michael Brant-Zawadzki ${ }^{1}$, Junko Hara ${ }^{2}$, Dennis Fortier ${ }^{3}$, Dori Holnagel ${ }^{1}$, Celine Keeble ${ }^{1}$, Nichole Guillen Nguyen ${ }^{4}$, Denise Macias ${ }^{1},{ }^{1}$ Hoag Neurosciences Institute, Newport Beach, California, United States, ${ }^{2}$ Shankle Clinic, Newport Beach, California, United States; ${ }^{3}$ Medical Care Corporation, Newport Beach, California, United States; ${ }^{4}$ Hoag Memorial Hospital Presbyterian, Newport Beach, California, United States.

Background: With the passage of the National Alzheimer's Prevention Act, the national focus on affordable healthcare solutions for the population at risk for Alzheimer's has become clear. A key element of that strategy should be to accelerate adoption of best-practice clinical standards identified during the past decade of intense research. Doing so will ensure that physicians are trained to identify early warning signs and intervene appropriately when patients present with a memory concern. The Orange County Vital Aging Program (OCVAP: www.OCVitalAging.org) is an effort to coordinate community resources and impose proactive principles of managing brain health across the at-risk population and the medical community that cares for it. Methods: The OCVAP has implemented 8 key program components: 1) PR/educational campaign; 2) web site with self-assessment tools for the public; 3) community cognitive screening services; 4) cognitive assessment tools for physicians; 5) network of community physicians trained to deliver proper ADRD healthcare; 6) expert physician panel guiding the broad physician community; 7) physician liaison to support community medical practices in adopting clinical guidelines, and 8) outcome analyses. Results: Within the first 18 months (as of 12/ $31 / 2011$ ), 15 educational seminars were attended by more than 1000 community members, $8 \mathrm{CME}$ courses educated more than 250 physicians, and more than 3000 self-assessments were completed online. In addition, the community screening services have attracted 1098 participants and found $24 \%$ prevalence of cognitive impairment (consistent with national estimates). Given evolving needs, we have created clinical guidelines for community physicians and links to physician support services and resources. An important aspect of the program is guiding participants with positive screening results to a well-trained physician in the community. As such, we recently strengthened the follow-up protocol with these participants and deployed a physician liaison to support the process. Conclusions: Changing public and physician behavior to achieve a more proactive approach to ADRD healthcare is a substantial, yet achievable challenge that requires a well-coordinated effort among a variety of medical and non-medical professionals, institutions, community-based organizations, and public advocates. Successful implementation of the OCVAP may provide a replicable model for other communities.

\section{O2-02-06 ALZHEIMER'S DISEASE INTERNATIONAL'S EFFORTS TO MAKE DEMENTIA A GLOBAL HEALTH PRIORITY}

Marc Wortmann, Alzheimer's Disease International, London, United Kingdom. 